\title{
Structure of a Bmi-1-Ring1B Polycomb Group Ubiquitin Ligase Complex*
}

Received for publication, March 16, 2006, and in revised form, May 10, 2006 Published, JBC Papers in Press, May 18, 2006, DOI 10.1074/jbc.M602461200

Zhizhong $\mathrm{Li}^{\ddagger 1}$, Ru Cao ${ }^{\S}$, Ming Wang ${ }^{\ddagger}$, Michael P. Myers ${ }^{\ddagger}$, Yi Zhang ${ }^{\S 2}$, and Rui-Ming Xu ${ }^{\ddagger 1,3}$

From the ${ }^{\ddagger}$ Cold Spring Harbor Laboratory, Cold Spring Harbor, New York 11724 and the ${ }^{\S}$ Howard Hughes Medical Institute and Department of Biochemistry and Biophysics, Lineberger Comprehensive Cancer Center, University of North Carolina, Chapel Hill, North Carolina 27599-7295

Polycomb group proteins $\mathrm{Bmi}-1$ and Ring1B are core subunits of the PRC1 complex, which plays important roles in the regulation of Hox gene expression, $\mathrm{X}$-chromosome inactivation, tumorigenesis, and stem cell self-renewal. The RING finger protein Ring1B is an E3 ligase that participates in the ubiquitination of lysine 119 of histone $\mathrm{H} 2 \mathrm{~A}$, and the binding of Bmi-1 stimulates the $\mathrm{E} 3$ ligase activity. We have mapped the regions of Bmi-1 and Ring1B required for efficient ubiquitin transfer and determined a 2.5- $\AA$ structure of the Bmi-1-Ring1B core domain complex. The structure reveals that Ring1B "hugs" Bmi-1 through extensive RING domain contacts and its $\mathrm{N}$-terminal tail wraps around Bmi-1. The two regions of interaction have a synergistic effect on the E3 ligase activity. Our analyses suggest a model where the Bmi-1-Ring1B complex stabilizes the interaction between the E2 enzyme and the nucleosomal substrate to allow efficient ubiquitin transfer.

Polycomb group proteins are a set of evolutionarily conserved transcriptional repressors controlling homeotic gene expression during development (1). Biochemical and genetic characterizations of Polycomb group proteins have revealed that they exist in distinct complexes, of which the two best characterized are the PRC1 ${ }^{4}$ and PRC2 complexes $(2,3)$. The Drosophila PRC1 core complex consists of Polycomb, Posterior Sex Combs, Polyhomeotic, and a Ring finger protein (4), and the mammalian complex contains homologous proteins (5). The PRC2 complex is a histone methyltransferase complex

\footnotetext{
* This work was supported in part by the W. M. Keck foundation (to R.-M. X.), the Howard Hughes Medical Institute (to Y. Z.), and National Institutes of Health Grants GM63718 (to R.-M. X.) and GM68804 (to Y. Z.). The costs of publication of this article were defrayed in part by the payment of page charges. This article must therefore be hereby marked "advertisement" in accordance with 18 U.S.C. Section 1734 solely to indicate this fact.

The atomic coordinates and structure factors (code 2HOD) have been deposited in the Protein Data Bank, Research Collaboratory for Structural Bioinformatics, Rutgers University, New Brunswick, NJ (http://www.rcsb.org/).

${ }^{1}$ Present address: Structural Biology Program, Skirball Institute of Biomolecular Medicine and Dept. of Pharmacology, New York University School of Medicine, New York, NY 10016.

${ }^{2}$ An Investigator of the Howard Hughes Medical Institute.

${ }^{3}$ To whom correspondence should be addressed (present address): Structural Biology Program, Skirball Institute of Biomolecular Medicine, New York University School of Medicine, 540 First Ave., New York, NY 10016. Tel.: 212-263-0585; Fax: 212-263-2150; E-mail: rmxu@saturn.med.nyu.edu.

${ }^{4}$ The abbreviations used are: PRC1, Polycomb repressive complex 1 ; E3, ubiquitin-protein isopeptide ligase; E2, ubiquitin carrier protein; GST, glutathione S-transferase; MALDI-TOF, matrix-assisted laser desorption ionization time-of-flight.
}

This is an Open Access article under the CC BY license. methylating lysine 27 of histone H3 (6-9). Both Polycomb group complexes have been implicated in diverse biological processes such as epigenetic inheritance, stem cell development, senescence, and tumorigenesis (10-12).

The PRC1 complex has at least two biochemical functions. One of which is to bind chromatin and prevent it from being remodeled by ATP-dependent remodeling factors (13). Using an electron microscope, a Drosophila PRC1 sub-complex has been seen to compact nucleosome arrays in vitro (14). A recent study revealed that a human PRC1 complex composed of Bmi-1, HPH2, PC3, and Ring proteins (Ring1A and Ring1B), which are homologs of Drosophila Posterior Sex Combs, Polyhomeotic, Polycomb, and dRing, respectively, is an E3 ubiquitin ligase complex that mono-ubiquitinates lysine 119 of nucleosomal histone H2A (15). The catalytic subunit of the PRC1 E3 ligase complex is Ring1B. The E3 ligase activity has been shown to be important for the involvement of PRC1 in X-chromosome inactivation and the control of Hox gene expression (16-19).

Very recently the mammalian PRC1 complex has been reconstituted using recombinant proteins (19). It was shown that Bmi-1, a Drosophila Posterior Sex Combs homolog that was originally discovered through its ability to collaborate with Myc in lymphomagenesis (20-22), plays a central role in the assembly of the PRC1 complex and, whereas Bmi-1 displays no detectable ubiquitin ligase activity, the binding of Bmi-1 greatly stimulates the E3 ligase activity of Ring1B $(15,19)$. To gain mechanistic insights into the assembly and enzymatic activity of the PRC1 complex, we have determined the structural requirements for the Bmi-1-Ring1B interaction and the basis for stimulation of the E3 ligase activity.

\section{MATERIALS AND METHODS}

Protein Expression and Purification-Human Ring1B and a Bmi-1 fragment encompassing residues 1-230 were overexpressed in Escherichia coli as an untagged and a GST fusion protein, respectively. A pCDFDuet-1 vector (Novagen) was used for the overexpression of Ring1B (cloned between the NdeI and XhoI sites). Bmi-1 was produced with a pGEX-KG-6P vector (Amersham Biosciences) carrying a preScission protease (Amersham Biosciences) cleavage site between the GST tag and the protein encoded by the cDNA fragment, which was cloned between the BamHI and Sall sites. Point mutants of Bmi-1 (T41R and D72R) and Ring1B (R70D) were generated by PCR and verified by DNA sequencing. The two proteins were coexpressed in the BL21(DE3) strain of E. coli. Both the wild-type and mutant Bmi-1-Ring1B complexes were first purified on a 
glutathione-Sepharose column. The GST tag was then removed by in-column digestion with the preScission protease, and the eluted Bmi-1-Ring1B complex was further purified by hydrophobic and gel-filtration column chromatography using a phenyl-Sepharose and a Superdex-200 (Amersham Biosciences) column, respectively.

Domain Mapping and Mass Analysis-The Bmi-1-Ring1B complex eluted from the glutathione-Sepharose column was subject to treatment with elastase at an $\sim 1: 100(\mathrm{w} / \mathrm{w})$ enzyme to protein ratio for $2 \mathrm{~h}$ at room temperature. The stable Bmi-1Ring1B core was then purified using the same procedure described above. The identities of the elastase-digested products were analyzed by mass spectrometry according to the following procedures: the samples was diluted directly into MALDI matrix (a saturated solution of 3-indole acrylic acid in $50 \%$ acetonitrile, $0.1 \%$ acetic acid) and spotted directly onto the MALDI plate. Spectra were acquired using an ABI 4700 mass spectrometer in linear mode. Another aliquot of sample was reduced and alkylated using TCEP and iodoacetamide and then digested with trypsin for $6 \mathrm{~h}$ in $50 \mathrm{~mm}$ diammonium phosphate $(\mathrm{pH}$ 8.0). Following digestion the sample was desalted using micro reverse phase columns, mixed with MALDI matrix, and spotted directly onto the MALDI plate. Spectra were acquired using the ABI 4700 , and matching peptides were confirmed by tandem mass spectrometry. The resulting data were analyzed using the MASCOT search engine.

Ubiquitin E3 Ligase Assay-Oligonucleosomes $(5 \mu \mathrm{g})$ were incubated with recombinant proteins in a 40- $\mu$ l reaction containing $50 \mathrm{~mm}$ Tris- $\mathrm{HCl}$ (pH 7.9), $5 \mathrm{~mm} \mathrm{MgCl}_{2}, 2 \mathrm{~mm} \mathrm{NaF}, 0.6$ mM dithiothreitol, $2 \mathrm{~mm}$ ATP, $10 \mu \mathrm{m}$ okadaic acid, $0.1 \mu \mathrm{g}$ of ubiquitin activation enzyme E1 (Calbiochem), $0.6 \mu \mathrm{g}$ of ubiquitin-conjugating enzyme Ubc5c, $1 \mu \mathrm{g}$ of FLAG-ubiquitin (Sigma). After incubation at $37^{\circ} \mathrm{C}$ for $1 \mathrm{~h}$, reaction was terminated by the addition of SDS-PAGE loading buffer. The proteins were resolved in $8-15 \%$ SDS-PAGE and blotted with anti-FLAG antibody.

Crystallization and Structure Determination-The elastasetreated mini Bmi-1-Ring1B core was concentrated to $\sim 20$ $\mathrm{mg} / \mathrm{ml}$ in a buffer containing $20 \mathrm{~mm}$ Tris at $\mathrm{pH} 8.0,500 \mathrm{~mm}$ $\mathrm{NaCl}, 1 \mathrm{~mm}$ EDTA, and $1.5 \mathrm{~mm}$ dithiothreitol for crystallization. The protein complex was crystallized by hanging-drop vapor diffusion in $0.1 \mathrm{M}$ Hepes ( $\mathrm{pH} 7.5$ ) and $20 \%$ (w/v) polyethylene glycol 8000 . The crystal belongs to the $\mathrm{P}_{3}$ space group with cell dimensions of $\mathrm{a}=\mathrm{b}=120.3 \AA$ and $\mathrm{c}=27.2 \AA$. X-ray absorption spectra at the zinc edge revealed the presence of zinc ions in the crystal, and three sets of zinc multiwavelength anomalous dispersion diffraction data were collected at $100 \mathrm{~K}$ at the X25 beamline of the National Synchrotron Light Source at Brookhaven National Laboratory. Data were processed using the HKL program (23), and statistics are shown in Table 1.

The structure was solved by the multiwavelength anomalous dispersion method. The positions of three zinc ions were located using the SOLVE program (24). Phasing was carried out using the PHASES program suite (25), and an additional zinc ion was identified using difference Fourier analysis. Model building was carried out using the graphic program O. Refinement was initially carried out using REFMAC (26) of the CCP4 program suite (27) and the statistics are shown in Table 1 . The

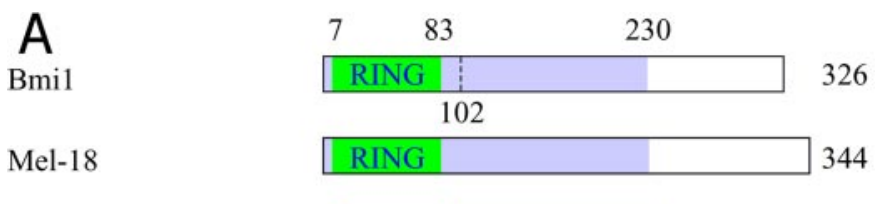

\begin{tabular}{ll|} 
hMBLR & RING \\
\cline { 2 - 2 }
\end{tabular}

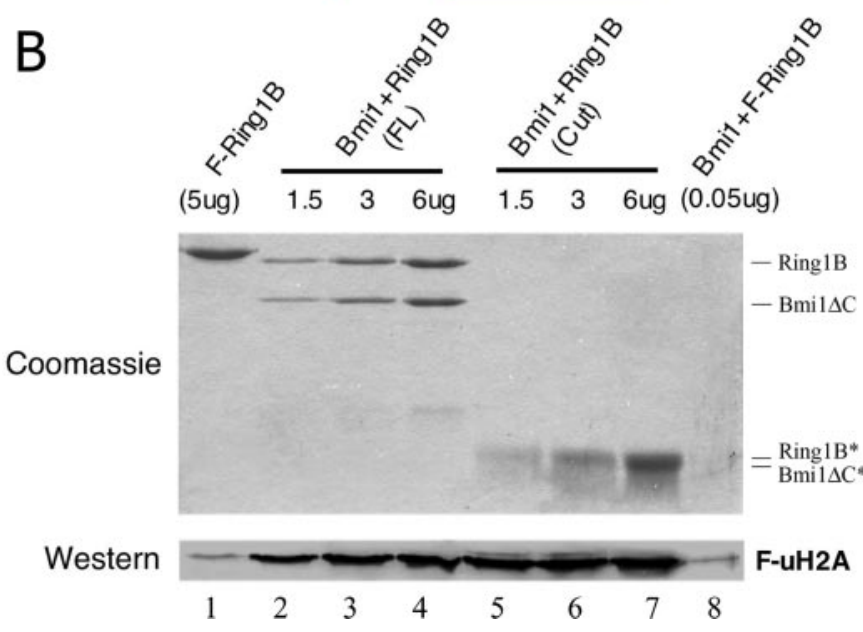

FIGURE 1. Functional domains of PRC1 proteins. $A$, a schematic representation of protein domains in Bmi-1, Mel-18, and MBLR. The conserved regions among the three proteins are gray, and the RING domains are green. The numbers indicate residues at the domain boundaries, and the dashed line indicates an elastase cleavage site. $B$, ubiquitin ligase activity assay. Top panel: Coomassie-stained SDS-PAGE gel showing Ring1B or Bmi-1-Ring1B samples used for the assay. The amount of protein used is indicated above each lane. Lane 1, FLAG-tagged full-length Ring1B expressed in bacteria. Lanes 2-4, increasing amounts of the heterodimeric complex of Bmi $1 \Delta \mathrm{C}$, which includes residues 1-230, with the full-length Ring1B. Lanes 5 and 6 , increasing amounts of elastase-digested products of the Bmi1-(1-230)-Ring1B complex. Lane 8, heterodimeric complex of full-length Bmi-1 and FLAG-tagged fulllength Ring1B expressed in Sf9 cells. Bottom panel: Western blot against the FLAG tag to detect the incorporation of FLAG-ubiquitin into histone H2A for each samples shown in the top panel.

figures were prepared with the programs PYMOL (28) and GRASP (29). The PDB accession code is $2 \mathrm{HOD}$.

\section{RESULTS}

Characterization of Bmi-1-Ring1B Interaction and Ubiquitin Ligase Activity-We first sought to determine the region of Bmi-1 that interacts with Ring1B. Because three mammalian Posterior Sex Combs homologs, Bmi-1, Mel-18, and MBLR, share significant sequence homology within the $\sim 230$ residue $\mathrm{N}$-terminal region (Fig. $1 A$ ), we reasoned that this region of Bmi-1 might be involved in interaction with Ring1B. To test this idea, we co-expressed full-length Ring1B with GST-tagged Bmi1-(1-230) in E. coli. The two proteins associate with each other throughout the purification process, which includes affinity purification using glutathione-agarose resins at $0.5 \mathrm{M}$ salt concentration as well as hydrophobic and gel-filtration column chromatography. As shown in lanes 2-4 of Fig. $1 B$ (top panel), the two proteins appear to be in a 1:1 complex at the end of the purification process. Furthermore, the Ring1B-Bmi1-(1230) complex has a significantly elevated ubiquitin ligase activity compared with Ring1B alone (Fig. $1 B$, compare lane 1 with lanes 2-4 of the bottom panel). At equimolar amounts of Ring1B, we estimate that the Ring1B-Bmi1-(1-230) complex is $\sim 25$-fold more active than Ring1B alone. In comparison, the 
Structure of a Bmi-1-Ring1B Complex

full-length Bmi-1-Ring1B complex expressed in insect cells is $\sim 100$-fold more active than that of equimolar Ring1B (Fig. $1 B$, lane 8). Thus, we conclude that Bmi1-(1-230) binds Ring1B and contains essential elements for stimulating the E3 ligase activity of Ring1B.

To further define the domains required for both Bmi-1Ring1B interactions and the E3 ligase activity, we carried out proteolytic mapping of the Ring1B-Bmi1-(1-230) complex. Digestion with elastase resulted in a stable band of $\sim 12 \mathrm{kDa}$ (Fig. 1B, top panel, lanes 5-7). Analyses using MALDI-TOF and TOF/TOF mass spectrometry revealed that the $\sim 12-\mathrm{kDa}$ band is a heterodimer of a $\mathrm{Bmi}-1$ fragment encompassing residues 1-102 and a Ring1B fragment encompassing residues 5-115. The elastase-processed complex of Bmi1-(1-102) and Ring1B(5-115), which we refer to as the mini Bmi-1-Ring1B complex, is stable and can be further purified through column chromatography. More importantly, the purified mini complex exhibits a level of ubiquitin ligase activity comparable to that of the Ring1B-Bmi1-(1-230) complex (Fig. 1B, bottom panel, lanes 5-7). Because Bmi-1 alone has no E3 ligase activity $(15,19)$, we thus conclude that Ring1B-(5-115) encompasses the region required for the histone $\mathrm{H} 2 \mathrm{~A}$ monoubiquitination E3 ligase activity, and Bmi1-(1-102) is sufficient for the stable interaction with Ring1B and stimulation of its E3 ligase activity.

Structure of the Bmi-1-Ring1B Mini Complex-To reveal the mechanism by which Bmi-1 binds to and stimulates the ubiquitin ligase activity of Ring1B at atomic resolution, we determined the crystal structure of the Bmi1-(1-102)-Ring1B-(5115) binary complex. The structure was determined to $2.5 \AA$ by the multiwavelength anomalous dispersion method using anomalous scattering signals from zinc ions that were co-purified and crystallized with the proteins. The refined structure has an $R$-factor of $21 \%$ and $R$-free of $25 \%$. The structure also has good stereochemical properties, because $90.4 \%$ of the residues are in the most favored region and none in the disallowed region of the Ramachandran plot calculated using PROCHECK (30). Detailed statistics of the crystallographic analyses are shown in Table 1.

The structure shows that both Bmi1-(1-102) and Ring1B(5-115) contain a canonical RING domain, which is composed of a three-stranded antiparallel $\beta$-sheet in the $2 \uparrow 1 \downarrow 3 \uparrow$ configuration, two zinc binding loops, and an $\alpha$-helix $(\alpha \mathrm{B})$ immediately $\mathrm{C}$-terminal to $\beta 2$ (Fig. $2, A$ and $B$ ). The above secondary structure elements, together with a short helix $(\alpha \mathrm{A})$ at the $\mathrm{N}$-terminal end (a helical turn in Ring1B) and a C-terminal helix $(\alpha \mathrm{C})$, constitute the RING domains of Bmi-1 and Ring1B. In each protein, the first zinc binding site is composed of a CXXC motif from the first zinc binding loop (L1) and another $\mathrm{CXXC}$ motif from $\alpha \mathrm{B}$. The second zinc binding site is composed of a $C X X C$ motif from the second zinc binding loop (L2) and a $\mathrm{CXH}$ motif located in $\beta 2$. The RING domains of Bmi-1 and Ring1B are quite similar: they share $\sim 30 \%$ sequence identity and $\sim 45 \%$ similarity. The two domains can be superimposed with a root-mean-squared deviation of $0.9 \AA$ using the $\mathrm{C} \alpha$ atoms of 61 of the 70 Ring1B RING domain residues for alignment with the corresponding $\mathrm{C} \alpha$ positions of $\mathrm{Bmi}-1$. In addition to the RING domain, Bmi1-(1-102) contains a 16-residue helix $(\alpha \mathrm{D})$ at the $\mathrm{C}$ terminus that projects away from the

\section{TABLE 1}

Summary of crystallographic analysis

\begin{tabular}{|c|c|c|c|}
\hline Data sets & Zinc inflection & Zinc peak & Zinc remote \\
\hline Wavelength $(\AA)$ & 1.2826 & 1.2821 & 1.2500 \\
\hline Resolution $(\AA)$ & 2.5 & 2.5 & 2.7 \\
\hline Measured reflections & 70,123 & 75,185 & 63,706 \\
\hline Unique reflections & 8,143 & 7,967 & 6,490 \\
\hline Average $I / \sigma$ & 15.9 & 21.6 & 18.8 \\
\hline Completeness $(\%, I / \sigma>0)$ & $96.9(94.2)$ & $96.8(100)$ & $96.6(95.2)$ \\
\hline$R_{\text {merge }}(\%)^{a}$ & $6.6(25.8)$ & $7.5(16.5)$ & $7.7(25.2)$ \\
\hline Phasing power (iso/ano) ${ }^{b}$ & $\mathrm{NA} / 1.54$ & $\mathrm{NA} / 2.45$ & $1.26 / 1.52$ \\
\hline Overall figure of merit & 0.49 & & \\
\hline $\begin{array}{l}\text { Refinement } \\
\text { Resolution range }(\AA) \\
R \text {-factor } / R_{\text {free }}(\%)^{c} \\
\text { No. of protein atom } \\
\text { No. of zinc ions } \\
\text { No. of water molecules }\end{array}$ & $\begin{array}{c}104.0-2.5 \\
21.0 / 24.7 \\
1,589 \\
4 \\
42\end{array}$ & & \\
\hline $\begin{array}{l}\text { Root mean square deviation } \\
\text { Bond lengths }(\AA) \\
\text { Bond angles }\left(^{\circ}\right)\end{array}$ & $\begin{array}{l}0.01 \\
1.33\end{array}$ & & \\
\hline $\begin{array}{l}{ }^{a} R_{\text {merge }}=\Sigma|I-\langle I\rangle| / \Sigma\langle I\rangle \text {, whe } \\
\text { measurements of the same re } \\
\text { reflections. } \\
{ }^{b} \text { Phasing power = room mean s } \\
\text { factor of the heavy atoms, and } \\
{ }^{c} R \text {-factor }=\Sigma\left|F_{o}\right|-\left|F_{c}\right| / \Sigma\left|F_{o}\right|, \\
\text { amplitude and } F_{c} \text { denotes the }\end{array}$ & $\begin{array}{l}I \text { and }\langle I\rangle \text { are the a } \\
\text { ection. The summa } \\
\text { are }\left(\left\langle F_{\mathrm{H}}\right\rangle / E\right) \text {, where } \\
\text { is the residual lack } \\
\text { vhere } F_{o} \text { denotes } \mathrm{t} \\
\text { ructure factor calcu }\end{array}$ & $\begin{array}{l}\text { averaged inten } \\
\text { ation is over } \\
F_{\mathrm{H}} \text { is the calc } \\
\text { of closure. } \\
\text { the observed } \\
\text { lated from th }\end{array}$ & $\begin{array}{l}\text { sity of multiple } \\
\text { all the observed } \\
\text { ulated structure } \\
\text { structure factor } \\
\text { e model. }\end{array}$ \\
\hline
\end{tabular}

body of the RING domain, whereas Ring1B-(5-115) has a 25-residue N-terminal tail that wraps around Bmi-1 (Fig. 2, $A$ and $B)$.

The extensive interaction between Bmi1-(1-102) and Ring1B-(5-115) buries a total pairwise surface area of $2459 \AA^{2}$. The two RING domains are closely juxtaposed and related by an approximate 2 -fold rotation (Fig. 2A). They pack against each other through a mixture of hydrophobic and polar interactions involving residues located on the central $\beta$-sheet, as well as, the $\mathrm{N}$ - and $\mathrm{C}$-terminal helices $(\alpha \mathrm{A}$ and $\alpha \mathrm{C})$ of both proteins (Fig. $2 C$ ). The packing of the RING domains contributes to the burial of a total surface area of $1055 \AA^{2}$. In addition, the long $\mathrm{N}$-terminal tail of Ring1B makes extensive interaction with the C-terminal helix, $\alpha \mathrm{D}$, and with the surface area of Bmi-1 opposite to the interface between the RING domains (Fig. 2, $A, B$, and $D$ ). The interaction between the $\mathrm{N}$-terminal tail of Ring1B and Bmi-1 buries a surface area of $1361 \AA^{2}$.

Structure-based Biochemical Analyses of the E3 Ligase Activity-Ideally we would like to quantitatively correlate the Bmi-1-Ring1B interaction with the E3 ligase activity of the binary complex using separately purified Bmi-1 and Ring1B wild-type or mutants proteins. However, this is not possible because each protein aggregates when purified separately and they do not form a binary complex efficiently when mixed together. Thus, we sought to introduce mutations that selectively perturb the interaction in different regions without completely disrupting the complex formation; hence the complexes were prepared by co-expression. Because both the RING domain and the N-terminal tail of Ring1B interact with Bmi-1, we reasoned that, if the interactions at both regions are important for the E3 ligase activity, a simultaneous change of interactions involving both regions would have a greater effect than those to each region individually. To alter the interaction 

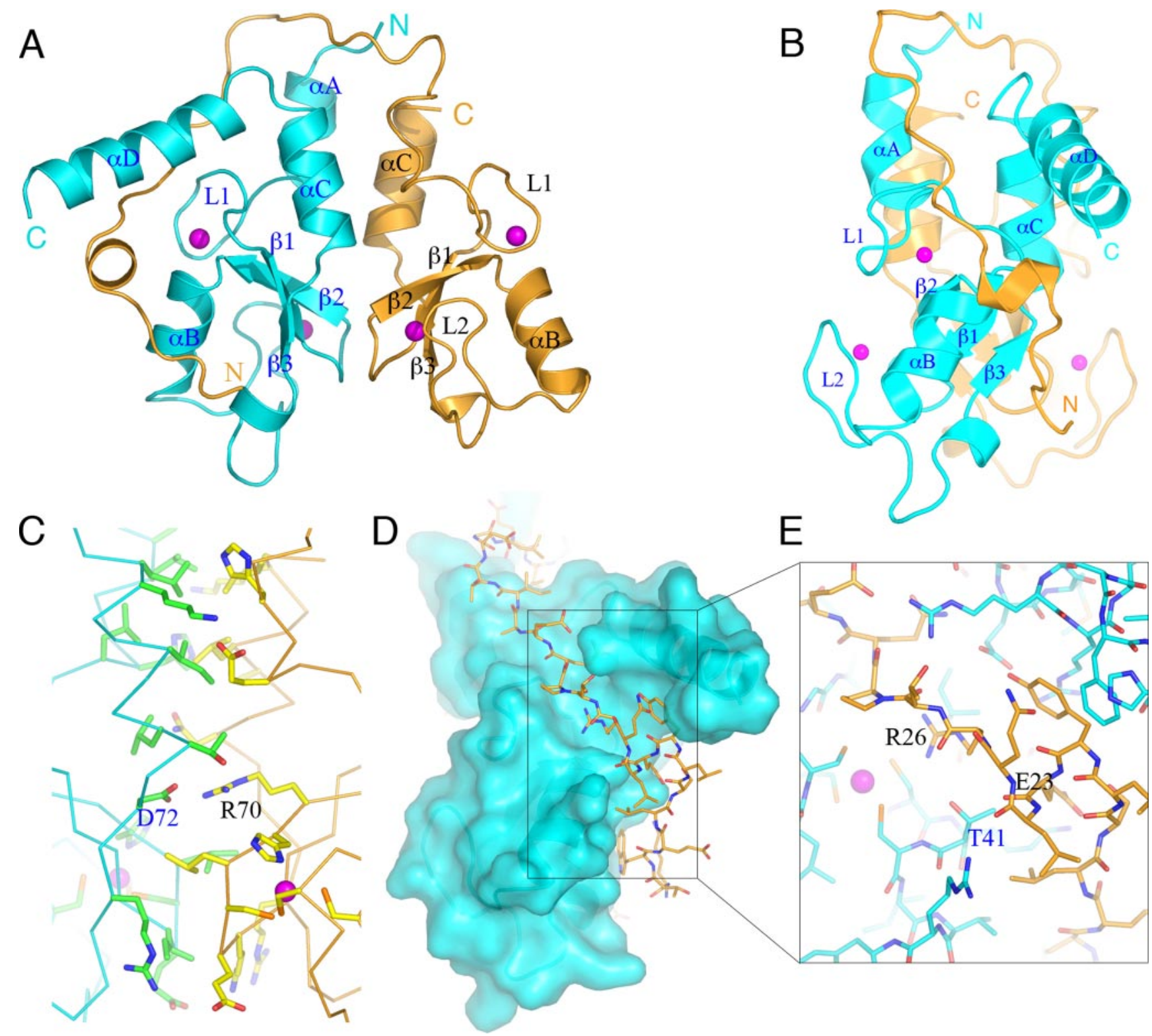

FIGURE 2. Structure of the mini Bmi-1-Ring1B complex. $A$, a ribbon representation of the heterodimer structure. Bmi-1 and Ring1B are shown in cyan and orange, respectively. Zinc ions are shown as magenta spheres. Main secondary structure elements are labeled. $B$, a view of the heterodimer from the left side with respect to the view direction in A. C, the interface of Bmi-1 and Ring1B RING domains involves a mixture of hydrophobic and polar interactions. The residues involved are shown in a stick model (carbon: green for Bmi-1 and yellow for Ring1B; nitrogen: blue; oxygen: red; sulfur: orange) superimposed with the Ca chains (cyan, Bmi-1; orange, Ring1B). A salt bridge involving Asp-72 of Bmi-1 and Arg-70 of Ring1B is labeled. D, the N-terminal tail of Ring1B wraps around $B m i-1$, which is shown in a surface representation and Ring1B in a stick model. The structure is viewed from a direction similar to that in $B$. The boxed area is shown in a close-in view in $E$. Thr-41 of Bmi-1 is buried in interactions with Glu-23 and Arg-26, and these residues are labeled.

between the N-terminal tail of Ring1B with Bmi-1 we changed Thr41 of Bmi-1 to an arginine (T41R), because it occupies a central position in the interaction with Ring1B through contacting both Glu-23 and Arg-26 (Fig. 2E). As shown in lanes 1 and 4 of Fig. 3 (bottom panel), the T41R mutant of Bmi1-(1230) still forms a complex with Ring1B, and the mutant complex exhibits a level of E3 ligase activity similar to that of the wild-type complex (Fig. 3, lanes 1 and 4 of the top panel). The T41R mutant of Bmi1-(1-230) is used as a negative control (Fig. 3 , top panel, lane 3), because Bmi-1 alone is not known to possess the E3 ligase activity, and the insect cell expressed fulllength Bmi-1-Ring1B complex and the mini Bmi-1-Ring1B complex are shown for comparison of E3 activity levels (Fig. 3,
Ctrl and lane 2 of the top panel). The T41R result suggests that changing the Bmi-1-Ring1B interaction at the $\mathrm{N}$-terminal tail region of Ring1 $\mathrm{B}$ alone does not cause detectable changes of the E3 ligase activity.

We next changed Asp-72 of Bmi-1, which is located in the RING domain and forms a salt bridge with Arg-70 of Ring1B in the middle of the RING domain interface (Fig. 2B), to an arginine (D72R). Similar to the T41R mutation, the D72R mutant complex also had an E3 ligase activity level similar to that of the wild-type complex (Fig. 3, lane 5). Because Asp-72 of Bmi-1 interacts with Arg-70 of Ring1B via a salt bridge, we made an R70D mutant of Ring1B and tested the degree to which it affects the E3 ligase activity. The Ring1B R70D mutant complex with 


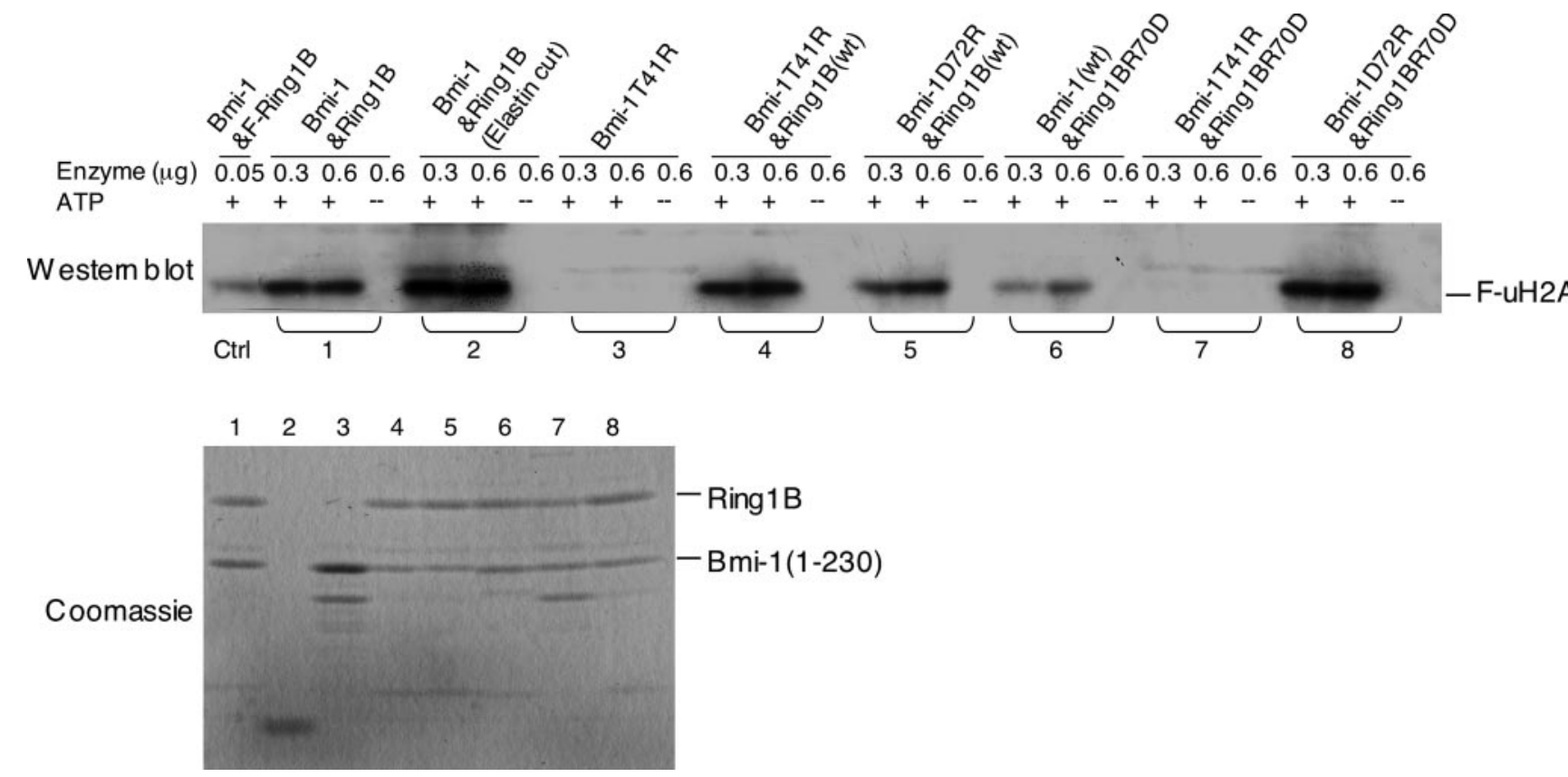

FIGURE 3. E3 ligase activity of Bmi-1-Ring1B complexes. Top panel: Western blot against FLAG-ubiquitin incorporated into histone H2A. The control lane (Ctrl) was with the full-length Bmi-1-Ring1B complex expressed in Sf9 cells. Lane 1: Bmi1-(1-230)-Ring1B assayed in three conditions indicated above the lanes. Lane 2, the mini Bmi-1-Ring1B complex derived from elastase digestion. Lane 3, the T41R mutant of Bmi1-(1-230). Lane 4, the T41R mutant of Bmi1-(1-230) in complex with Ring1B. Lane 5, the D72R mutant of Bmi1-(1-230) in complex with Ring1B. Lane 6, wild-type Bmi1-(1-230) in complex with the R70D mutant of Ring1B. Lane 7, the T41R mutant of Bmi1-(1-230) in complex with the R70D mutant of Ring1B. Lane 8, the D72R mutant of Bmi-1 with the R70D mutant of Ring1B. The three assay conditions used for lanes $1-8$ are: 0.3 and $0.6 \mu \mathrm{g}$ of the indicated proteins and in the absence of ATP, respectively. Bottom panel: Coomassie-stained SDS-PAGE gel showing the protein samples used for the E3 ligase activity assay shown in lanes 1-8 of the top panel.

the wild-type Bmi1-(1-230) exhibits reduced enzymatic activity in comparison with the wild-type complex (Fig. 3, lane 6). Thus, the two residues involved in the salt-bridge interaction appear to affect the E3 ligase activity of the heterodimeric complex to different degrees.

A dramatic reduction of the E3 ligase activity is displayed by the complex of the T41R mutant of Bmi1-(1-230) and the R70D mutant of Ring1B (Fig. 3, lane 7), which changes the interaction between Bmi-1 and Ring1B in both contacting regions. It should be pointed out that the T41R-R70D complex is noticeably less stable than other Bmi-1-Ring1B complexes used in this study, because a significant fraction of the mutant complex falls apart during the purification process. A contaminant band co-purified with Bmi1-(1-230) also co-purifies with the mutant complex (Fig. 3, lanes 3 and 7 of the bottom panel). It is possible that the sample shown in lane 7 is a mixture of the mutant protein complex and uncomplexed Bmi-1. The apparent instability of the T41R-R70D complex and the lack of detectable E3 ligase activity (Fig. 3, lane 7 of the upper panel) confirms our hypothesis that both of the Bmi-1-Ring1B interaction regions identified from our structural study are important for the E3 ligase activity.

We next sought to restore the interaction between the two proteins by combining the R70D mutant of Ring1B with the D72R mutant of Bmi-1, because this combination should restore the salt-bridge interaction. Indeed, the mutant complex of Ring1B R70D and Bmi-1 D72R rescues the reduction of enzymatic activity caused by the R70D mutation of Ring1B (Fig. 3, lane 6) and displays a wild-type E3 ligase activity level (Fig. 3, lane 8). Given the practical difficulty of quantitatively measuring the binding between Bmi-1 and Ring1B using separately purified proteins, the above mutagenesis and binding data reveal a positive correlation between stable Bmi-1-Ring1B interaction and the E3 ligase activity of the binary complex.

\section{DISCUSSION}

We have biochemically and structurally characterized the Bmi-1 and Ring1B domains required for both a stable interaction and stimulation of the E3 ligase activity. The structure revealed that Ring1B interacts with Bmi-1 via both the RING domain and the extended N-terminal tail. Comparing the structure of the mini Bmi-1-Ring1B complex with the only other heterodimeric RING domain structure, the BRCA1BARD1 RING-RING complex (31), reveals that the two RING domains in the Bmi-1-Ring1B complex are positioned in a fashion similar to that of the BRCA1-BARD1 complex (Fig. $4 A$ ), although detailed conformations and residues mediating the intermolecular interactions differ between the two structures. It is likely that the observed packing of RING domains represent a general mode of interaction between RING domains. Interestingly, the RING domain of BRCA1 also has in vitro E3 ligase activity, and the binding of BARD stimulates the E3 activity of BRCA1, although the physiological substrates remain elusive $(32,33)$. The most significant differences between the two structures are between the $\mathrm{N}$-terminal regions of Ring1B and BRCA1 and the C-terminal regions of Bmil and BARD, both of which are involved in intermolecular interactions (Fig. 4A).

Previous structural studies have indicated that E3 ligases may function to position the substrate and the E2 enzyme in a stable configuration that allows efficient transfer of ubiquitin. The structure of the RING finger E3 ligase c-Cbl in complex with its E2 enzyme, UbcH7, shows that the E2 enzyme binds to a region 


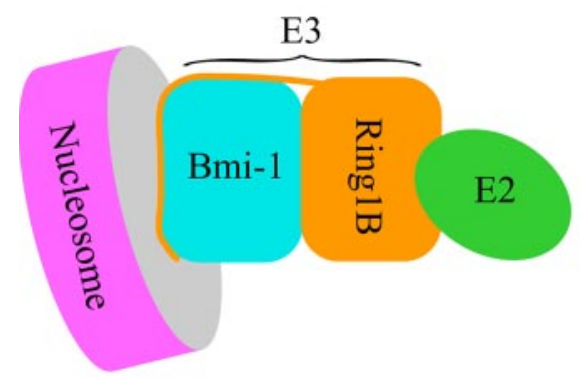

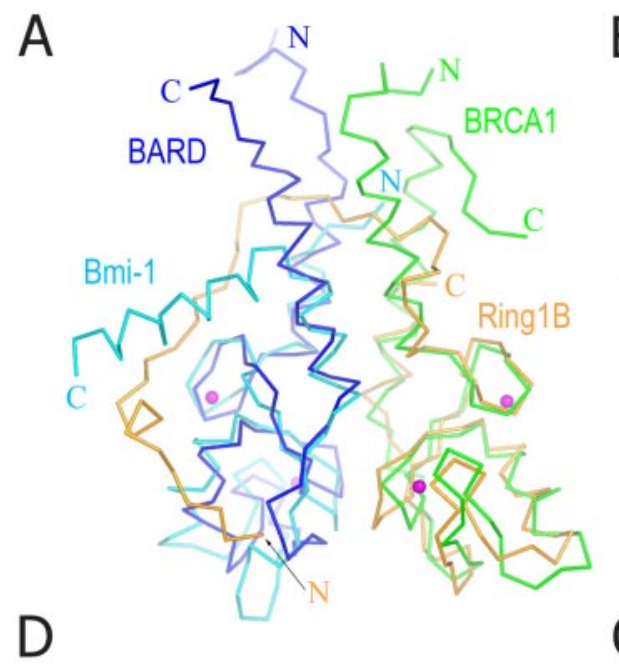
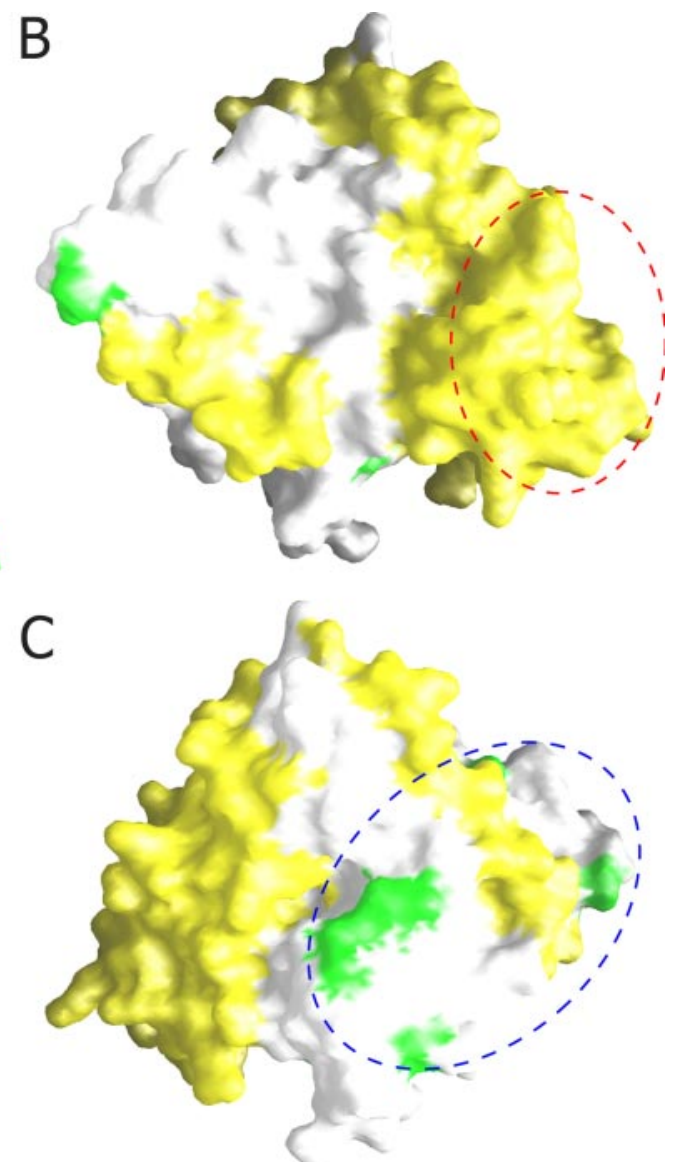

FIGURE 4. Structural comparison and modeling. $A$, superposition of the mini Bmi1-Ring1B complex with the BRCA1-BARD RING domain complex. The Ca chains of Bmi-1, Ring1B, BRCA1, and BARD are colored cyan, orange, green, and blue, respectively. The $\mathrm{N}$ and $\mathrm{C}$ termini of each chain are labeled with letters of the corresponding color. $B$, a surface representation of the mini Bmi-1-Ring1B complex with the surface of Ring1B colored yellow and Bmi-1 in white (left panel). The green surface patches indicate the positions occupied by residues that differ between Bmi-1 and Mel-18. The area enclosed in the red dotted ellipse indicates the implicated E2 enzyme binding site. $C$, the same protein surfaces viewed from the back with respect to the position in $B$. The area enclosed in the blue dotted ellipse is enriched with residues different between Bmi- 1 and Mel-18, and the area may represent a substrate binding site. $D$, a schematic diagram showing a model for the $\mathrm{E} 3$ ligase function of the Bmi-1-Ring1B complex where the heterodimeric complex facilitates an efficient transfer by position the substrate and the E2 enzyme in an optimal configuration.

spanning the two zinc-binding loops and the intervening helix ( $\alpha \mathrm{B}$ in our nomenclature) of the RING domain of c-Cbl (34). Interestingly, the E2-E3 interactions appear to be structurally conserved. The $\mathrm{E} 3$ binding sequences of $\mathrm{UbcH} 7$ and $\mathrm{UbcH} 5$ are highly similar, which allows us to infer the $\mathrm{E} 2$ binding site of the Bmi-1-Ring1B mini complex using the c-Cbl-UbcH7 structure as a reference. As seen in Fig. $4 B$, the E2 binding site is mapped to the Ring1B surface encompassing the two Zinc binding loops and $\alpha \mathrm{B}$, which is not involved in Bmi-1-Ring1B interactions. The corresponding region of the BRCA1 RING domain has been demonstrated to bind $\mathrm{UbcH} 5$, which further supports the idea that this region of Ring1B binds the E2 enzyme (35).

How does the binding of Bmi-1 stimulate the E3 ligase activity? There are two possibilities: 1 ) the binding of $\mathrm{Bmi}-1$ may relieve the inhibitory effect of a part of Ring1B, such as the $\mathrm{N}$-terminal tail; 2) Bmi-1 may play a more active role in the chemical transfer of ubiquitin. The first scenario is unlikely because our mutagenesis data show that the E3 ligase activity is lost even when the two proteins are still associated with each other in the Bmi-1 T41R and Ring1B R70D combination, whereas the R70D mutant of Ring1B alone has a reduced but respectable E3 ligase activity. Another support of our reasoning is that Mel-18, a Bmi-1 homo$\log$ that only differs by seven residues within the regions of interest here (and none of these regions are located in the interface with Ring1B), lacks the ability to stimulate the E3 ligase activity even though it interacts with Ring1B (19). A more likely scenario is that Bmi-1 actively promotes ubiquitination through binding the substrate. An analysis of the distribution of the residues different between Bmi- 1 and Mel- 18 shows that they are enriched in a region distant from the implicated E2 binding site (Fig. 4C), which suggests that the different ability of Bmi-1 and Mel-18 in stimulating the E3 ligase activity of Ring1B is not due to direct involvements with E2 binding but rather in substrate binding. Interestingly, a portion of the $\mathrm{N}$-terminal tail is located within the putative substrate binding region (Fig. 4C), making it likely that this part of Ring1B participates in the positioning of nucleosomal substrates together with Bmi-1.

It is interesting to note that the proposed binding sites for the nucleosomal substrate and the E2 enzyme are far apart. In fact this phenomenon is exactly what has been implicated in previous structural studies (36). Although E3 enzymes differ widely, a consensus theme of their function is emerging, which is to provide a scaffold for positioning the E2 enzyme and the substrate $(36,37)$. Combining the knowledge from previous studies with our current analyses, a model of the Bmi-1-Ring1B E3 ligase function is depicted in Fig. 4D, which provides a basis for further in-depth studies of nucleosomal substrate specificity and mechanisms of E3 ligase activation of the PRC1 complex.

Acknowledgments-We thank Michael Becker for help at the X25 beamline of National Synchrotron Light Source and Lee Henry for comments on the manuscript.

\section{REFERENCES}

1. Ringrose, L., and Paro, R. (2004) Annu. Rev. Genet. 38, 413-443

2. Francis, N. J., and Kingston, R. E. (2001) Nat. Rev. Mol. Cell. Biol. 2, 409-421

3. Simon, J. A., and Tamkun, J. W. (2002) Curr. Opin. Genet. Dev. 12, 210-218

4. Francis, N. J., Saurin, A. J., Shao, Z., and Kingston, R. E. (2001) Mol. Cell 8, 545-556

5. Levine, S. S., Weiss, A., Erdjument-Bromage, H., Shao, Z., Tempst, P., and 


\section{Structure of a Bmi-1-Ring1B Complex}

Kingston, R. E. (2002) Mol. Cell. Biol. 22, 6070-6078

6. Cao, R., Wang, L., Wang, H., Xia, L., Erdjument-Bromage, H., Tempst, P., Jones, R. S., and Zhang, Y. (2002) Science 298, 1039-1043

7. Czermin, B., Melfi, R., McCabe, D., Seitz, V., Imhof, A., and Pirrotta, V. (2002) Cell 111, 185-196

8. Kuzmichev, A., Nishioka, K., Erdjument-Bromage, H., Tempst, P., and Reinberg, D. (2002) Genes Dev. 16, 2893-2905

9. Muller, J., Hart, C. M., Francis, N. J., Vargas, M. L., Sengupta, A., Wild, B., Miller, E. L., O'Connor, M. B., Kingston, R. E., and Simon, J. A. (2002) Cell 111, 197-208

10. Valk-Lingbeek, M. E., Bruggeman, S. W., and van Lohuizen, M. (2004) Cell 118, 409-418

11. Gil, J., Bernard, D., and Peters, G. (2005) DNA Cell Biol. 24, 117-125

12. Cao, R., and Zhang, Y. (2004) Curr. Opin. Genet. Dev. 14, 155-164

13. Shao, Z., Raible, F., Mollaaghababa, R., Guyon, J. R., Wu, C. T., Bender, W., and Kingston, R. E. (1999) Cell 98, 37-46

14. Francis, N. J., Kingston, R. E., and Woodcock, C. L. (2004) Science 306, $1574-1577$

15. Wang, H., Wang, L., Erdjument-Bromage, H., Vidal, M., Tempst, P., Jones, R. S., and Zhang, Y. (2004) Nature 431, 873-878

16. Fang, J., Chen, T., Chadwick, B., Li, E., and Zhang, Y. (2004) J. Biol. Chem. 279, 52812-52815

17. de Napoles, M., Mermoud, J. E., Wakao, R., Tang, Y. A., Endoh, M., Appanah, R., Nesterova, T. B., Silva, J., Otte, A. P., Vidal, M., Koseki, H., and Brockdorff, N. (2004) Dev. Cell 7, 663-676

18. Hernandez-Munoz, I., Lund, A. H., van der Stoop, P., Boutsma, E., Muijrers, I., Verhoeven, E., Nusinow, D. A., Panning, B., Marahrens, Y., and van Lohuizen, M. (2005) Proc. Natl. Acad. Sci. U. S. A. 102, 7635-7640

19. Cao, R., Tsukada, Y. I., and Zhang, Y. (2005) Mol. Cell 20, 845-854

20. van Lohuizen, M., Verbeek, S., Scheijen, B., Wientjens, E., van der Gulden, H., and Berns, A. (1991) Cell 65, 737-752
21. Haupt, Y., Alexander, W. S., Barri, G., Klinken, S. P., and Adams, J. M. (1991) Cell 65, 753-763

22. Brunk, B. P., Martin, E. C., and Adler, P. N. (1991) Nature 353, 351-353

23. Otwinowski, Z., and Minor, W. (1997) Method Enzymol. 276, 307-326

24. Terwilliger, T. C., and Berendzen, J. (1999) Acta Crystallogr. D 55, $849-861$

25. Furey, W., and Swaminathan, S. (1997) Method Enzymol. 277, 590-620

26. Murshudov, G. N., Vagin, A. A., and Dodson, E. J. (1997) Acta Crystallogr. Sect. D Biol. Crystallogr. 53, 240-255

27. CCP4 (1994) Acta Crystallogr. Sect. D Biol. Crystallogr. 50, 760-763

28. DeLano, W. L. (2002) The PYMOL Molecular Graphics System, DeLano Scientific, San Carlos, CA

29. Nicholls, A., Sharp, K. A., and Honig, B. (1991) Proteins 11, 281-296

30. Laskowski, R. A., MacArthur, M. W., Moss, D. S., and Thornton, J. M. (1993) J. Appl. Crystallogr. 24, 946-956

31. Brzovic, P. S., Rajagopal, P., Hoyt, D. W., King, M. C., and Klevit, R. E. (2001) Nat. Struct. Biol. 8, 833-837

32. Hashizume, R., Fukuda, M., Maeda, I., Nishikawa, H., Oyake, D., Yabuki, Y., Ogata, H., and Ohta, T. (2001) J. Biol. Chem. 276, 14537-14540

33. Xia, Y., Pao, G. M., Chen, H. W., Verma, I. M., and Hunter, T. (2003) J. Biol. Chem. 278, 5255-5263

34. Zheng, N., Wang, P., Jeffrey, P. D., and Pavletich, N. P. (2000) Cell 102, 533-539

35. Brzovic, P. S., Keeffe, J. R., Nishikawa, H., Miyamoto, K., Fox, D., 3rd, Fukuda, M., Ohta, T., and Klevit, R. (2003) Proc. Natl. Acad. Sci. U. S. A. 100, 5646-5651

36. Zheng, N., Schulman, B. A., Song, L., Miller, J. J., Jeffrey, P. D., Wang, P., Chu, C., Koepp, D. M., Elledge, S. J., Pagano, M., Conaway, R. C., Conaway, J. W., Harper, J. W., and Pavletich, N. P. (2002) Nature 416, 703-709

37. Reverter, D., and Lima, C. D. (2005) Nature 435, 687-692 